\title{
How Metallic Impurities in Carbon Cathodes Affect the Electrochemistry of Aluminum Batteries
}

\author{
Jasmin Smajic ${ }^{*[a]}$, Filipa R. Fernandes Simoes ${ }^{[a]}$, and Pedro M. F. J. Costa*[a]
}

[a] J. Smajic, F. R. F. Simoes, Prof. P. M. F. J. Costa

Physical Science and Engineering Division

King Abdullah University of Science and Technology (KAUST)

Thuwal 23955-6900, Saudi Arabia

E-mail: jasmin.smajic@kaust.edu.sa; pedro.dacosta@kaust.edu.sa

Supporting information for this article is given via a link at the end of the document.

\begin{abstract}
As a promising electrochemical energy storage system, rechargeable aluminum batteries face critical challenges in their quest for commercial viability. While the design of suitable cathodes has attracted much attention, their chemical composition and purity has been less of a concern. This is especially true for carbon cathodes, where the presence of metallic impurities is often overlooked. Herein, we demonstrate the influence that transition metals exert on the electrochemistry of carbon nanotube cathodes for non-aqueous aluminum batteries. In the presence of chloroaluminate electrolytes, these synthesis by-products originate inflated capacities, increased self-discharge and misleading electrochemical signatures, among others. Thus, our findings affirm the need for strict control of the composition and purity of materials (and components) used in nonaqueous aluminum batteries.
\end{abstract}

Aluminum batteries have gained attention as promising low cost and sustainable energy storage devices. ${ }^{[1]}$ Amongst the numerous systems proposed, coupling carbon cathodes with aluminum plate anodes has emerged as the favorite setup for rechargeable Al batteries (AIB). ${ }^{[2]}$ Adding to this, the preferred electrolytes are, currently, non-aqueous ionic liquids. Here, the most popular is 1-ethyl-3-methylimidazolium chloride ([EMIm]Cl) as mixing it with $\mathrm{AlCl}_{3}$ yields a melt that generates the electroactive ions $\mathrm{AlCl}_{4}^{-}$and $\mathrm{Al}_{2} \mathrm{Cl}_{7}^{-} .^{-[3]}$ Unfortunately, this ionic liquid (IL) is acidic. Often, it reacts with other battery components such as the current collector and cell casing (Figure S1) because, for the major part, these are made from transition metals and metal alloys (e.g. $\mathrm{Cu}, \mathrm{Ni}$, steel). ${ }^{[4]}$ Therefore, within the voltage operation window of nonaqueous AIB, it is desirable to avoid the contact of IL with other metals.

Knowingly, the key points of using carbon powders (e.g. graphite flakes and carbon filaments) as the cathode material of $([\mathrm{EMIm}] \mathrm{Cl})$-based systems is their resilience (chemical, mechanical and structural) and the easy insertion/de-insertion of $\mathrm{AlCl}_{4}{ }^{-}$into/from the graphitic particles. [2b, 3d, 5] However, depending on the synthesis conditions, these powders may contain a non-negligible amount of impurities. For instance, the bottom-up synthesis of graphene or carbon nanotubes by chemical vapor deposition relies on elemental catalysts. On the other hand, for a top-down process, such as the synthesis of reduced graphene oxide ( $\mathrm{rGO}$ ) or the mechanical exfoliation of graphite flakes, contaminants may be introduced via process steps (e.g. the Hummer's method) ${ }^{[6]}$ or be present in the graphite purchased from vendors (e.g. with geological origin, when mined, or from industrial processing, Kish flakes). ${ }^{[7]}$ Generally, these impurities are transition metals such as $\mathrm{Fe}, \mathrm{Co}, \mathrm{Ni}, \mathrm{Cu}$ and $\mathrm{Mo}$. Furthermore, the concentration of impurities can differ between batches of the same sample. ${ }^{[8]}$

Electrochemical techniques are extremely sensitive to the presence of elements that catalyze different reactions. ${ }^{[9]}$ In the literature, several reports identified trace levels of metals as the active components of catalytic systems whose carbon powders had been assumed "metal-free".[10] Whilst in electrocatalysis this issue has been discussed, the same cannot be said for the energy storage field wherein the presence of metallic impurities in carbons is overlooked and their contribution to the electrochemistry is often not acknowledged. Thus, it is not surprising that little attention has been given to how metallic impurities affect the electrochemical signature of Al batteries, this despite the predominant use of acidic chloroaluminate electrolytes. Here, we demonstrate the effect of having metallic impurities in carbon cathodes used for non-aqueous Al batteries. To do so, we employed two different, and commercially available, single-walled carbon nanotube (SWCNT) powders as the active electrode material that were studied before and after subjecting them to a purification process. We should clarify that we refer to the metallic catalysts presence as impurities, instead of dopants, because of their unrecognized contribution to the electrochemistry of carbon materials. [11] The two commercial samples of nanotubes were characterized as-received (see Supporting Information). Judging from our data and the information provided by the vendor, the SWCNT were synthesized by chemical vapor deposition (CVD). The assessment of metallic impurities was carried out with inductively-coupled plasma - optical emission spectroscopy (ICP-OES; Table 1 and Supporting Information). The nanotubes showing a predominance of Co and Mo are dubbed SWCNT-1 whereas those where $\mathrm{Fe}$ is the main metal element are labeled SWCNT-2. The purification was undertaken using acid reflux (Figure 1a and Supporting Information), a process that was optimized to remove the metal content without compromising the SWCNT structure (note: introducing lattice 
defects, or changing the nanotubes' aspect ratio, will alter their electrochemical signature).

a)

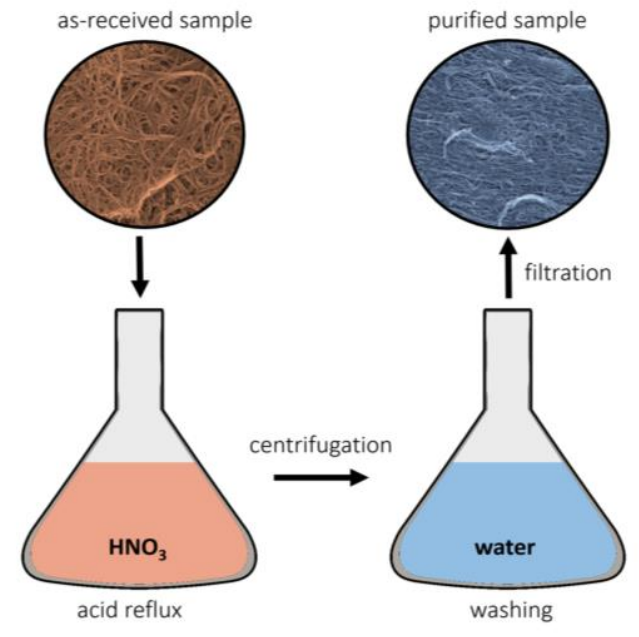

b)

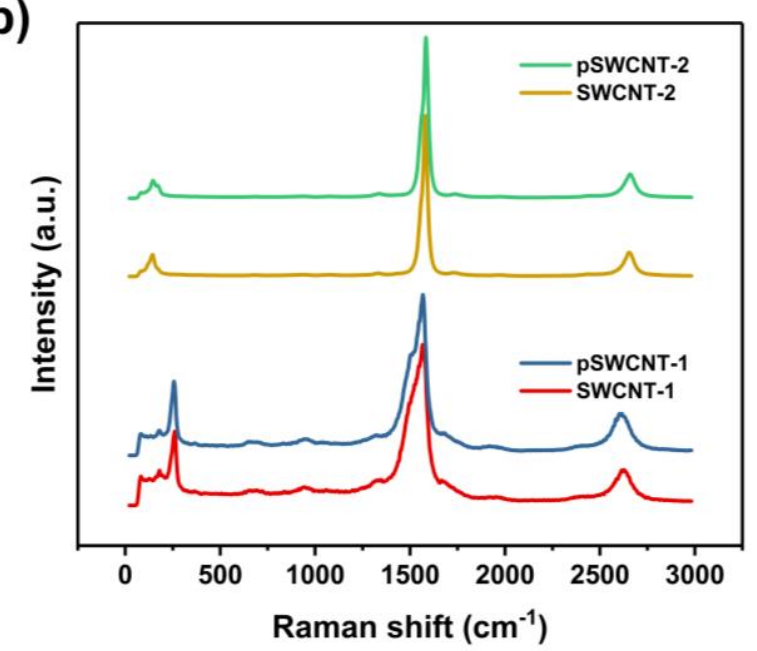

Figure 1. a) Schematic illustration of the purification of SWCNT samples; b) Raman spectra of the as-received and purified SWCNT-1 and SWCNT-2.

The structural integrity was confirmed with Raman spectroscopy (Figure 1b). For the two SWCNT, the ratio of the $D$ - to $G$-bands intensity was small $\left(I_{D} / I_{G} \leq 0.1\right)$ confirming their high structural quality and a general lack of defects, before and after the purification step. ${ }^{[12]}$ Hereafter, the purified nanotubes ( $p$ ) are labeled pSWCNT-1 and pSWCNT-2.

Table 1. Metallic impurities content (in ppm) of unpurified SWCNT-1 and SWCNT-2, and purified, pSWCNT-1 and pSWCNT-2, samples as determined by ICP-OES. LOD = limit of detection.

\begin{tabular}{|c|c|c|c|c|}
\hline Element & SWCNT-1 & pSWCNT-1 & SWCNT-2 & pSWCNT-2 \\
\hline Co & $\begin{array}{l}4245.1 \pm \\
192.0\end{array}$ & $556.2 \pm 5.3$ & $<L O D$ & $<L O D$ \\
\hline $\mathrm{Cr}$ & $\begin{array}{l}227.5 \pm \\
28.5\end{array}$ & $124.5 \pm 6.3$ & $435.9 \pm 2.1$ & $146.2 \pm 16.1$ \\
\hline $\mathrm{Fe}$ & $\begin{array}{l}511.8 \pm \\
57.4\end{array}$ & 221.326 .5 & $\begin{array}{l}93780.6 \pm \\
6513.3\end{array}$ & $\begin{array}{l}13138.2 \pm \\
698.9\end{array}$ \\
\hline Mn & $18.9 \pm 0.1$ & $<L O D$ & $38.8 \pm 1.4$ & $\angle L O D$ \\
\hline Mo & $\begin{array}{l}25831.4 \pm \\
1449.4\end{array}$ & $\begin{array}{l}4593.6 \pm \\
37.4\end{array}$ & $38.8 \pm 1.4$ & $<L O D$ \\
\hline $\mathbf{N i}$ & $56.8 \pm 0.4$ & $19.2 \pm 1.1$ & $842.9 \pm 16.9$ & $276.7 \pm 9.9$ \\
\hline
\end{tabular}

Looking in detail the chemical composition of the samples, it is clear that both contained a large content of metallic impurities (Table 1). In the as-received SWCNT-1, the concentrations found for Co and Mo are very high. The presence of these elements is, however, drastically reduced after purification: from 2.6 wt\% (25831.4 $\pm 1449.4 \mathrm{ppm})$ down to $0.5 \mathrm{wt} \%(4593.6 \pm 37.4 \mathrm{ppm})$ for Mo and from $0.4 \mathrm{wt} \%$ $(4245.1 \pm 192.0 \mathrm{ppm})$ to $0.05 \mathrm{wt} \%(556.2 \pm 5.3 \mathrm{ppm})$ for Co. With regards to the SWCNT-2, the main element was Fe, with roughly $9.4 \mathrm{wt} \%(93780.6 \pm 6513.3 \mathrm{ppm})$ present in the asreceived powder. Still, after the acid treatment, the tubes retain $1.3 \mathrm{wt} \%(13138.2 \pm 698.9 \mathrm{ppm})$ of Fe. Therefore, the purification method used was not entirely effective in removing the Fe impurity. ${ }^{[13]}$ In addition to these elements, we noticed the presence of other metals such as $\mathrm{Cr}, \mathrm{Mn}$ and $\mathrm{Ni}$. In all cases, their concentration was lower and got reduced to $<0.03$ wt\% after purification.

The electrochemical behavior of the nanotubes is summarized in Figure 2. The cyclic voltammograms of the as-received SWCNT-1 and purified pSWCNT-1 (Figure 2a) show an extreme difference in peak current intensities. This is especially noticeable below $0.6 \mathrm{~V}$ (Figure $2 \mathrm{a}$, inset) with the advent of a strong reduction wave for SWCNT-1, which is not present in the purified counterpart. For the SWCNT-2 and pSWCNT-2 powders (Figure $\mathbf{2 b}$ ) while the difference in peak current intensities is smaller, the clear redox pair at $0.2 \mathrm{~V}$ and $0.8 \mathrm{~V}$ in the as-received sample, disappears after purification. Correlating these results with the data sets from Raman spectroscopy (Figure 1b) and ICP-OES (Table 1) implies that the disparity in electrochemical responses is mainly due to the presence of metallic impurities, Co/Mo for SWCNT-1 and Fe for SWCNT-2. In order to assess the practical effect of these impurities on battery performance, we looked at the charge/discharge behavior of the nanotubes (Figure $\mathbf{2 c}$ ). The SWCNT-1 exhibits an unusually large capacity of $581 \mathrm{mAh} \mathrm{g}^{-1}$ that gradually decays and then stabilizes at $41 \mathrm{mAh} \mathrm{g}^{-1}$ (after 70 cycles). This capacity, as seen from Figure $\mathbf{2 d}$, arises from a clear plateau starting at $0.6 \mathrm{~V}$, which is in agreement with the cyclic voltammogram (Figure 2a). The pSWCNT-1, on the other hand, shows an initial discharge capacity of $29 \mathrm{mAh} \mathrm{g}^{-1}$ which rises slightly and then steadies at $49 \mathrm{mAh} \mathrm{g}^{-1}$, in latter cycles. This is accompanied by a lack of plateaus, further confirming the unambiguous effect of $\mathrm{Co} / \mathrm{Mo}$ impurities in the asreceived samples. As for the SWCNT-2 (Figure $2 \mathrm{c}$ ), it exhibited consistent capacities of around $30 \mathrm{mAh} \mathrm{g}^{-1}$ between the asreceived and purified states. Remarkably, the small discharge 
plateau at $0.2 \mathrm{~V}$ disappears completely after the Fe presence is reduced (Figure $\mathbf{2 d}$, inset). Also of note, all samples reach similar capacities after 70 cycles. Next, we looked at the Coulombic efficiencies (Figure S2). Both SWCNT-1 and pSWCNT-1 stabilize at $\sim 70 \%$, while SWCNT-2 and pSWCNT-2 do so at $\sim 100 \%$. Additionally, contaminants appear to decrease the open circuit potential (OCV) of the cells (Figure 2e). After charging the cells to $2.0 \mathrm{~V}$, the SWCNT-1 loses $\sim 1.1$
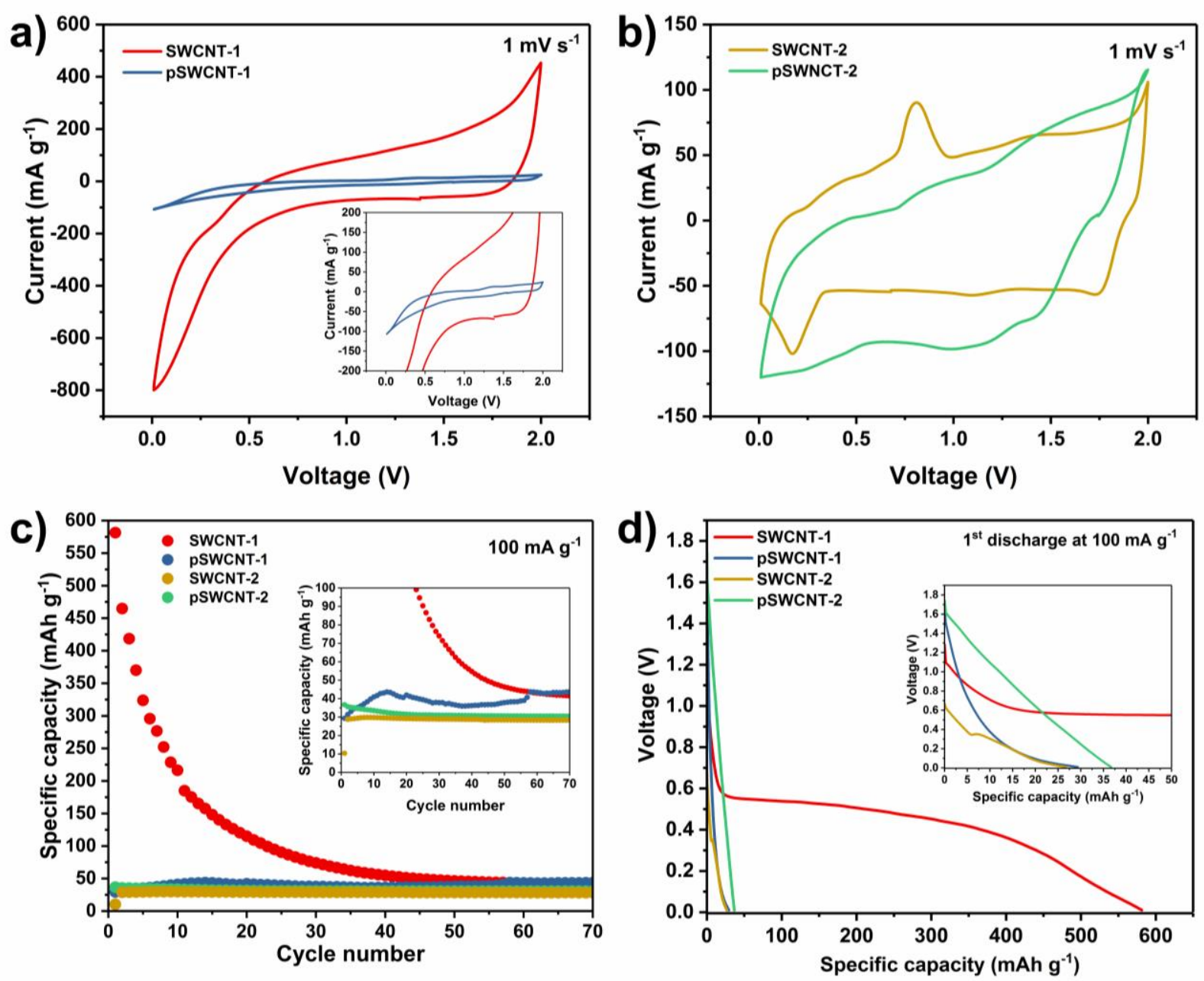

e)

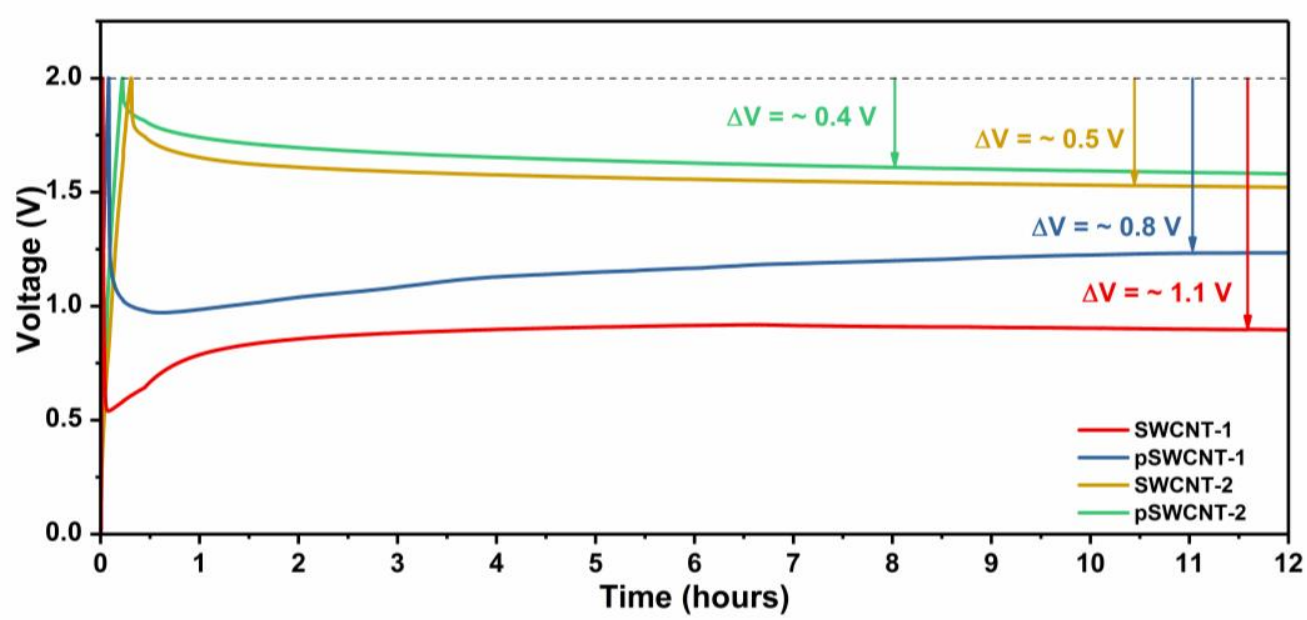

Figure 2. The electrochemical behavior of different SWCNT electrodes: a) cyclic voltammograms of SWCNT-1 and pSWCNT-1 cathodes (inset shows an amplified section); b) cyclic voltammograms of SWCNT-2 and pSWCNT-2 cathodes; c) discharge capacities (inset shows an amplified section); d) voltage profiles (inset shows an amplified section); e) OCV drop. 
Up to now, it is clear that the presence of impurities can dominate the electrochemical signature of carbon nanomaterials. For the SWCNT-1, Mo is a known inert current collector for $\mathrm{Al}$ batteries with $\mathrm{AlCl}_{3}:[\mathrm{EMIm}] \mathrm{Cl}$ electrolytes, therefore it is unlikely to explain this sample's electrochemical response. On the other hand, Co (and its oxides) have been extensively used in Al batteries, exhibiting discharge voltages of $0.6 \mathrm{~V}$, similar to what we observed. ${ }^{[14]}$ As for the SWCNT-2, $\mathrm{Fe}$ is an element prone to redox reactions. The effect of the metals was confirmed by analysing the as-received nanotubes and corresponding cycled slurries. Two localised elemental profiling methods, X-ray photoelectron spectroscopy (XPS) and scanning electron microscopy (SEM) - energy dispersive $X$-ray spectroscopy (EDX), were used because of their different spatial resolutions and penetration depths (Figures S3 and S4, respectively). While metallic impurities were always detected in the as-received SWCNTs, their spectral peak intensities were strongly diminished on the post-cycled slurries. Interestingly, the higher penetration depth of the focused electrons from SEM was necessary to identify some of those elements. The post-cycling intensity reduction is an indication that the redox-active transition metals were spent in irreversible electrochemical reactions. This would also account for the capacity decay and its subsequent stabilisation at values similar to those of the purified SWCNT samples.

To test the effect of $\mathrm{Co}$ and $\mathrm{Fe}$ in non-aqueous $\mathrm{Al}$ batteries, the halogens salts $\mathrm{CoCl}_{2}$ and $\mathrm{FeCl}_{2}$ were used as active cathode materials instead of the nanotubes. Knowing that $\mathrm{Cl}$ has an electronegativity of 3.16 (vs 1.88 for Co and 1.83 for Fe), the reduction wave of $\mathrm{CoCl}_{2}$ and $\mathrm{FeCl}_{2}$ is expected to be shifted towards more positive potential values compared to those of SWCNT-1 and SWCNT-2, where metals are most likely in their native form. ${ }^{[15]}$ This was confirmed by the linear sweep

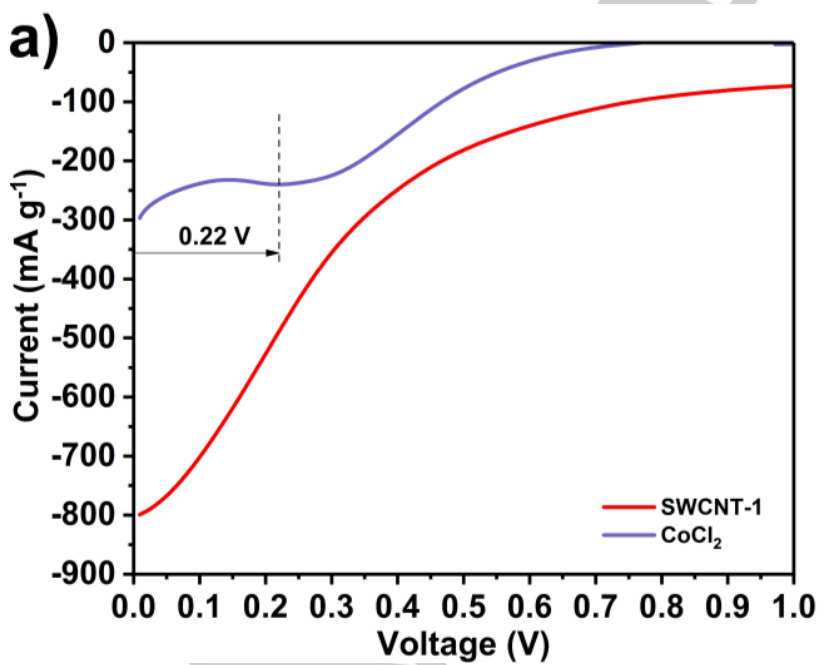

voltammetry presented in Figures $\mathbf{3 a}$ and $\mathbf{3 b}$. Both $\mathrm{CoCl}_{2}$ and $\mathrm{FeCl}_{2}$ show reduction peaks at values slightly higher $(0.22 \mathrm{~V}$ and $0.11 \mathrm{~V}$, respectively) than those for SWCNT-1 and SWCNT2. The $\mathrm{CoCl}_{2}$ reduction peak is centered at $0.22 \mathrm{~V}$, while the one for $\mathrm{FeCl}_{2}$ is situated at $0.28 \mathrm{~V}$, corroborating $\mathrm{Co}$ and $\mathrm{Fe}$ impurities as the reason for different electrochemical behavior of the as-received and purified samples. Lastly, it is important to note the disparity in peak reduction currents: $\mathrm{CoCl}_{2}$ exhibits an order of magnitude higher current intensity than $\mathrm{FeCl}_{2}$. This explains the order of magnitude higher capacity obtained for SWCNT-1 when compared to that of SWCNT-2. Such a result is all the more remarkable when considering the clear redox peaks in the CV of the latter (Figure 2b) as well as the much higher concentration of the electrochemically active transition metal in the SWCNT-2 powder $\left([\mathrm{Co}]_{\text {SWCNT }-1}=4245 \mathrm{ppm}\right.$ and $\left.[\mathrm{Fe}]_{\text {SWCNT }-2}=93781 \mathrm{ppm}\right)$. We assume this to be the consequence of the nanotubes' catalytic synthesis mechanisms. When employing $\mathrm{Fe}$ as the CVD catalyst, the metal particles get supersaturated with carbon and graphene layers get extruded. Upon cooling (or poisoning) of the process, the formation of $\mathrm{Fe}_{3} \mathrm{C}$ is expected. Commonly, post-synthesis Fe particles show graphitic layers that are higher in number and better structured than those of $\mathrm{Co} / \mathrm{Mo}$ catalytic systems. Altogether, the Fe will be more shielded and these particles will be less electrochemically active. ${ }^{[9 a}$ 16] This hypothesis concurs well with the observations from the spectroscopical analyses. The need to use higher energy focused electrons (SEM, $20 \mathrm{kV}$ ) to clearly identify Fe in the as-received SWCNT- 2 can only be explained through the shielding effect provided by a thick and wellstructured graphitic shell enveloping these particles (XPS, 1.5 $\mathrm{keV})$.

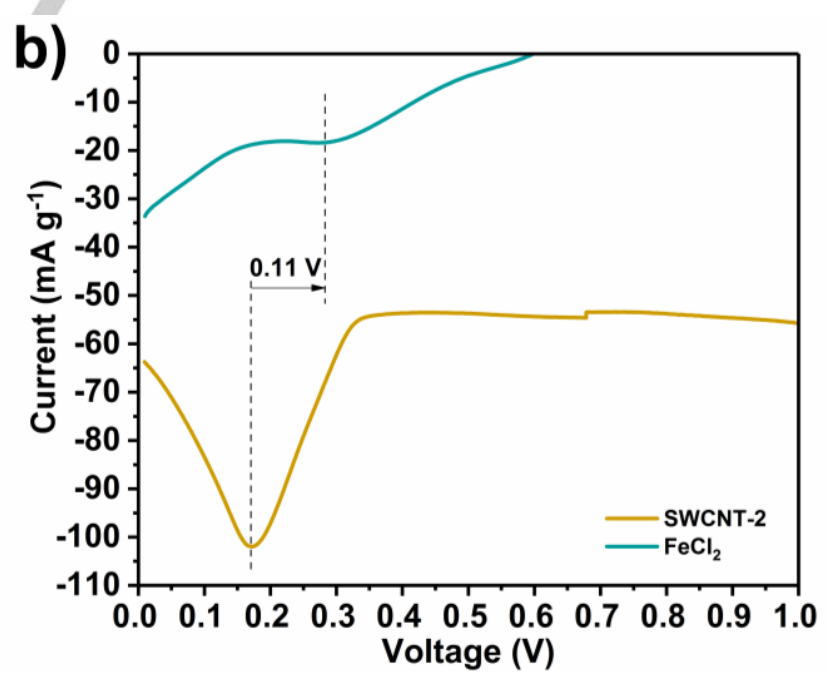

Figure 3. a) Linear sweep voltammetry of SWCNT-1 and $\mathrm{CoCl}_{2}$; b) linear sweep voltammetry of SWCNT-2 and $\mathrm{FeCl}_{2}$.

From the above, as-received commercial carbon materials may not be suitable for direct use in Al batteries with $\mathrm{AlCl}_{3}:[\mathrm{EMIm}] \mathrm{Cl}$ electrolytes. As shown, catalytic impurities had a profound effect on the electrochemical signature of nanotube powders. The transition metals directly participated in irreversible electrochemical reactions and were exhausted after several tens of cycles, tending to the intrinsic capacity of the pure SWCNT.

By affecting the electrochemical response, impurities may lead to incorrect conclusions. One example of how misleading this can be is exemplified by Al-S battery systems. ${ }^{[17]}$ In a recent study, ${ }^{[18]}$ our Al-S cells showed a discharge voltage 
plateau around $0.6 \mathrm{~V}$, just like Co does here. In these circumstances, it would be tempting for us to claim that all capacity came from $\mathrm{S}$ alone and that the SWCNT did not participate in the electrochemical reactions. In fact, this same argument was previously used by other authors. It was only after carrying out the purification of the nanotubes that we could assert the real contribution of sulphur. Beyond electrochemical processes, metal impurities could affect other studies such as impact in the environment and cell toxicity analysis. In an industrial setting, and if due care is not taken, the use of non-purified carbon materials for Al battery cathodes could result in unaccounted amounts of transition metals being handled in production lines. To make matters worse, the concentration of impurities could vary from batchto-batch. ${ }^{[8-9]}$ Hence, we believe that the assessment of the electrode's material purity, especially in the case of nanocarbons, must be done for each and every batch of sample with appropriate elemental analysis techniques. ${ }^{[19]}$ In conclusion, it should be understood that the use of the acidic $\mathrm{AlCl}_{3}:[\mathrm{EMIm}] \mathrm{Cl}$ electrolyte in $\mathrm{Al}$ battery systems necessitates strict purity control of the electrodes' materials, as well as the other cell components. Here, we demonstrated that as-received SWCNT can contain metal impurities which exert significant influence on a battery's electrochemical response. Sometimes unreported, bulk elemental analysis of the cathode material is paramount to avoid altered cyclic voltammograms, inflated capacities, inaccurate Coulombic efficiencies and increased self-discharge. Whilst exemplified for nanotubes, these conclusions should apply to other carbon-based electrode materials that are produced via catalytic (e.g. CVD), chemical (e.g. graphite oxidation/exfoliation) or industrial (e.g. Kish graphite) processes, all of which are known to include transition metals in their as-made powders.

\section{Acknowledgements}

This work was funded by KAUST (BAS/1/1346-01-01). The authors thank the KAUST Core Labs for technical assistance, in particular Dr. Nimer Wehbe for his help with X-ray photoelectron spectroscopy.

\section{Conflict of Interest}

The authors declase no conflict of interest.

Keywords: impurities $\cdot$ aluminum battery $\bullet$ carbon $\bullet$ ionic liquid $\bullet$ electrochemistry

[1] a) T. Leisegang, F. Meutzner, M. Zschornak, W. Munchgesang, R. Schmid, T. Nestler, R. A. Eremin, A. A Kabanov, V. A. Blatov, D. C. Meyer, Front. Chem. 2019, 7 , 268; b) D. Muñoz - Torrero, M. Anderson, J. Palma, R. Marcilla, E. Ventosa, ChemElectroChem 2019, 6, 27662770.

[2] a) Y. Zhang, S. Liu, Y. Ji, J. Ma, H. Yu, Adv. Mater. 2018 , 30, 1706310; b) M. C. Lin, M. Gong, B. Lu, Y. Wu, D. Y. Wang, M. Guan, M. Angell, C. Chen, J. Yang, B. J. Hwang, H. Dai, Nature 2015, 520, 325-328.
[3] a) N. Jayaprakash, S. K. Das, L. A. Archer, Chemical Communications 2011, 47, 12610-12612; b) H. Sun, W. Wang, Z. Yu, Y. Yuan, S. Wang, S. Jiao, Chem. Commun. 2015, 51, 11892-11895; c) X. G. Sun, Z. Bi, H. Liu, Y. Fang, C. A. Bridges, M. P. Paranthaman, S. Dai, G. M. Brown, Chem. Commun. 2016, 52, 1713-1716; d) C. Y. Chen, T. Tsuda, S. Kuwabata, C. L. Hussey, Chem. Commun. 2018, 54, 4164-4167.

[4] Y. Oh, G. Lee, Y. Tak, ChemElectroChem 2018, 5, 33483352.

[5] a) Y. X. Hu, S. Debnath, H. Hu, B. Luo, X. B. Zhu, S. C. Wang, M. Hankel, D. J. Searles, L. Z. Wang, J. Mater. Chem. A 2019, 7, 15123-15130; b) R. D. McKerracher, A. Holland, A. Cruden, R. G. A. Wills, Carbon 2019, 144, 333-341; c) J. Smajic, A. Alazmi, N. Batra, T. Palanisamy, D. H. Anjum, P. Costa, Small 2018, 14, 1803584; d) D. Y. Wang, C. Y. Wei, M. C. Lin, C. J. Pan, H. L. Chou, H. A. Chen, M. Gong, Y. Wu, C. Yuan, M. Angell, Y. J. Hsieh, Y. H. Chen, C. Y. Wen, C. W. Chen, B. J. Hwang, C. C. Chen, H. Dai, Nat. Commun. 2017, 8, 14283; e) H. Y. Hu, T. H. Cai, P. Bai, J. Xu, S. H. Ge, H. Hu, M. B. Wu, Q. Z. Xue, Y. F. Yan, X. L. Gao, W. Xing, Chem. Commun. 2020, 56, 1593-1596.

[6] A. Alazmi, S. Rasul, S. P. Patole, P. M. F. J. Costa, Polyhedron 2016, 116, 153-161.

[7] a) A. Ambrosi, S. Y. Chee, B. Khezri, R. D. Webster, Z Sofer, M. Pumera, Angew. Chem. 2012, 51, 500-503; b) J. Wang, J. Liu, H. Zhang, H. Zhang, Y. Li, Geol. J. 2020, 55 , 3748-3769; c) M. Kierkowicz, E. Pach, A. Santidrian, S. Sandoval, G. Goncalves, E. c) Tobias-Rossell, M. Kalbac, B. Ballesteros, G. Tobias, Carbon 2018, 139, 922-932.

[8] J. Kameník, F. R. F. Simões, P. M. F. J. Costa, J. Kučera, V. Havránek, J. Radioanal. Nucl. Chem. 2018, 318, 24632472.

[9] a) W. Kiciński, S. Dyjak, Carbon 2020, 168, 748-845; b) C Kim, Y.-K. Choe, D. H. Won, U. Lee, H.-S. Oh, D. K. Lee, C. H. Choi, S. Yoon, W. Kim, Y. J. Hwang, ACS Energy Lett. 2019, 4, 2343-2350; c) J. Zhang, Y. Liu, C. Sun, P. Xi, S. Peng, D. Gao, D. Xue, ACS Energy Lett. 2018, 3, 779-786; d) W. Q. Zaman, Z. Wang, W. Sun, Z. Zhou, M. Tariq, L. Cao, X.-Q. Gong, J. Yang, ACS Energy Lett. 2017, 2, 2786-2793.

[10] a) L. Wang, Z. Sofer, M. Pumera, Nanoscale 2019, 11, 11083-11085; b) L. Wang, A. Ambrosi, M. Pumera, Angew. Chem. 2013, 52, 13818-13821; c) M. Pumera, H. Iwai, Chem. - Asian J. 2009, 4, 554-560; d) M. Pumera, H Iwai, J. Phys. Chem. C 2009, 113, 4401-4405; e) M. Pumera, ACS Catal. 2020, 10, 7087-7092; f) L. Wang, Z. Sofer, M. Pumera, ACS Nano 2020, 14, 21-25.

[11] S. M. Tan, M. Pumera, ACS Nano 2019, 13, 2681-2728.

[12] J. Smajic, A. Alazmi, S. P. Patole, P. M. Costa, RSC Adv. 2017, 7, 39997-40004.

[13] M. Pumera, Langmuir 2007, 23, 6453-6458.

[14] a) J. Liu, Z. Y. Li, X. G. Huo, J. L. Li, J. Power Sources 2019, 422, 49-56; b) X. Xiao, M. Y. Wang, J. G. Tu, Y. W. Luo, S. Q. Jiao, ACS Sustainable Chem.\& Eng. 2019, 7, 16200-16208.

[15] J. Wang, W.-F. Lin, Y. Shi, H.-S. Wang, L.-Q. Rong, X.-H. Xia, Chem. Commun. 2016, 52, 13409-13412.

[16] C. P. Deck, K. Vecchio, Carbon 2006, 44, 267-275.

[17] a) H. Yang, L. Yin, J. Liang, Z. Sun, Y. Wang, H. Li, K. He, L. Ma, Z. Peng, S. Qiu, C. Sun, H. M. Cheng, F. Li, Angew. Chem. 2018, 57, 1898-1902; b) T. Gao, X. Li, X. Wang, J. Hu, F. Han, X. Fan, L. Suo, A. J. Pearse, S. B. Lee, G. W. Rubloff, K. J. Gaskell, M. Noked, C. Wang, Angew. Chem. 2016, 55, 9898-9901; c) Z. Hu, Y. Guo, H. Jin, H. Ji, L. J. Wan, Chem. Commun. 2020, 56, 20232026.

[18] J. Smajic, S. Wee, F. R. F. Simoes, M. N. Hedhili, N. Wehbe, E. Abou-Hamad, P. M. F. J. Costa, ACS Appl. Energy Mater. 2020, 3, 6805-6814.

[19] a) J. Shi, J. Zhang, J. Guo, ACS Energy Lett. 2019, 4, 2124-2129; b) K. V. Kravchyk, C. Seno, M. V. Kovalenko, ACS Energy Lett. 2020, 5, 545-549. 


\section{COMMUNICATION}

\section{Entry for the Table of Contents}

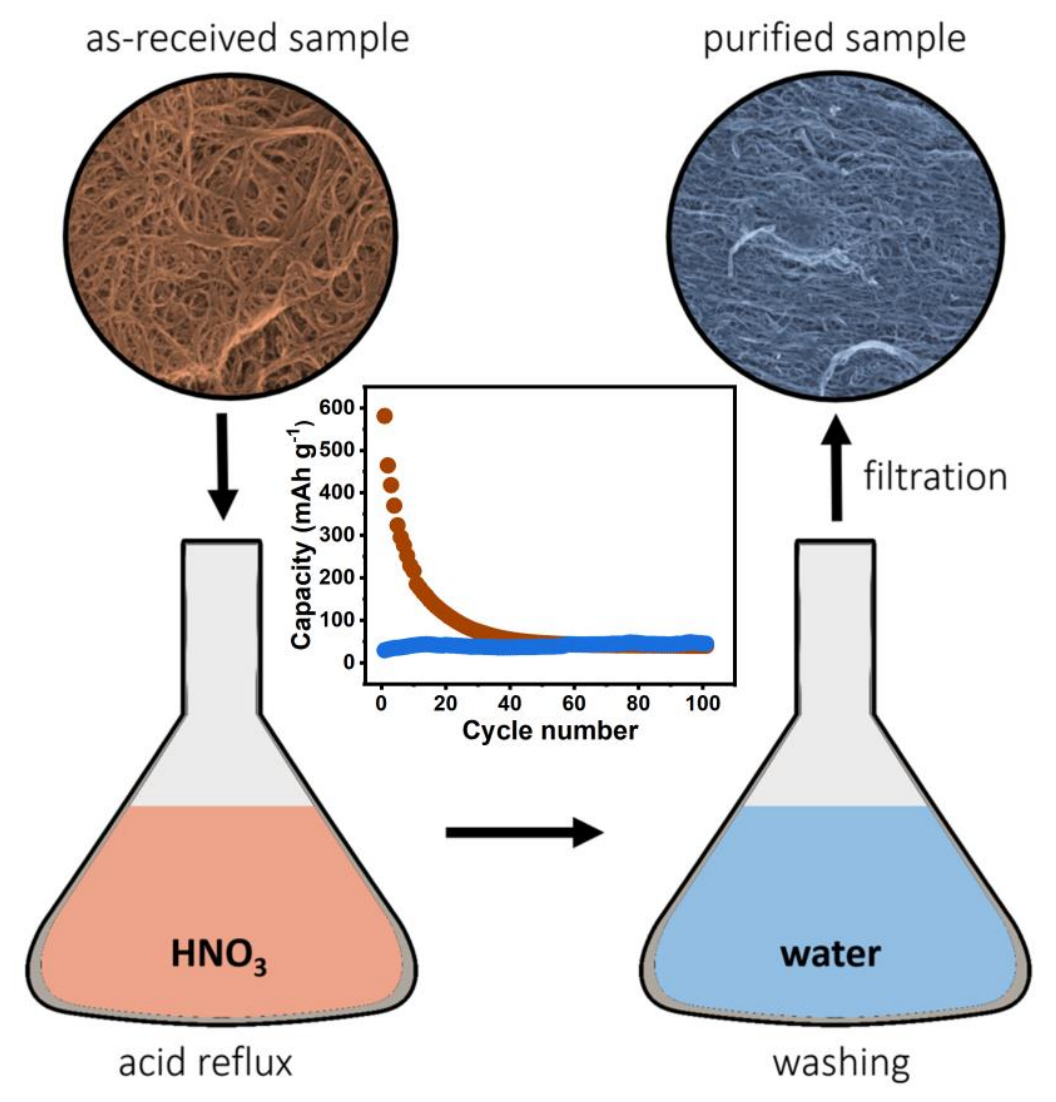

The need for strict control of the chemical composition of materials (and components) used in non-aqueous aluminum batteries is demonstrated by the notable effect that transition metal particles, present in non-purified carbon-based cathodes, have on the electrochemical signature, capacity and self-discharge of these systems.

Institute and/or researcher Twitter usernames: twitter.com/smajasmin 